Article

\title{
Friction Coefficient Compensation Control in Synchronizer Synchronization Process for Transmission
}

\author{
Hao Yan ${ }^{1}$, Zhaoping $\mathrm{Xu}^{1, * \mathbb{C}}$, Juntang Yuan ${ }^{1}$, Meiyan Liu ${ }^{2}$ and Wenqing $\mathrm{Ge}^{2}$ \\ 1 School of Mechanical Engineering, Nanjing University of Science and Technology, Nanjing 210094, China \\ 2 School of Transportation and Vehicle Engineering, Shandong University of Technology, Zibo 255000, China \\ * Correspondence: xuzhaoping@njust.edu.cn
}

Received: 15 July 2019; Accepted: 29 July 2019; Published: 31 July 2019

check for updates

\begin{abstract}
In the synchronization process of a synchronizer, the friction coefficient of the friction cone is changed because of friction heat. In this paper, the influence of a changing friction coefficient on shift quality was analyzed. The quantitative relationship between friction coefficient and its influencing factors was studied through a synchronizer synchronization process test bench. Based on the quantitative analysis and an optimal shift force control method, a compensation control strategy for friction coefficient was established. Moreover, the effectiveness of the compensation control was verified through simulation and experiment. The results showed that the friction coefficient was maintained near the expected value of 0.08 after the compensation control, the shifting speed difference the synchronous time was shortened by nearly $0.12 \mathrm{~s}$, and the sliding friction was reduced by $4.64 \mathrm{~J}$ under the experimental conditions. The analysis and compensation of the friction coefficient provide a theoretical reference for improving shift quality.
\end{abstract}

Keywords: lock-ring synchronizer; friction coefficient; compensation control; shift force; synchronization performance

\section{Introduction}

As one of the main components of a transmission, the synchronizer generates friction torque during friction synchronization, thereby eliminating the difference in rotational speed between the two ends of the synchronizer, to achieve shifting [1]. During the synchronization of the synchronizer, the friction coefficient of the friction cone is changed because of friction heat, which ultimately affects the shift control accuracy of the transmission [2].

A lot of basic research has been proposed to improve shift control accuracy $[3,4]$. The temperature changing regularities of the friction surface under different working conditions were analyzed by simulation and experiment $[5,6]$. The influence of temperature on the synchronizer's working performance was verified in literature [7]. The friction and heat generation process of the synchronizer with different friction coefficients were analyzed in the literature $[8,9]$. The results showed that the greater the friction coefficient is, within a certain range, the higher the temperature between the friction surfaces. Additionally, Li presented a model of predicting wear in the friction lining of a wet clutch and introduced that the wear mechanism is related to thermal degradation [10]. Marklund developed a friction model which takes temperature, speed, and nominal pressure into account for the wet clutch during boundary lubrication conditions [11]. The friction surface temperature and the friction coefficient of the friction cone are the critical influences on the shift quality, which need further study.

Currently, research on car synchronizers has mainly focused on the influence of the geometry of the synchronizer and the synchronous inertia on the synchronous performance, and considered 
the friction coefficient between the friction pairs of the synchronizer as a constant. In reference [12], the ADAMS software was used to establish the virtual prototype model of the lock-ring synchronizer, and the influence of each parameter on the shift synchronization time was studied. Huang Hua regarded the friction coefficient as a constant in the process of establishing the synchronizer model [13]. In the synchronous shift phase, the synchronizer realizes the synchronization function by sliding between the friction cones. The length of the synchronization time is directly determined by the friction coefficient between the friction cones, so the calculation method of considering the friction coefficient as a constant is not accurate. Besides this, with the deepening of the research on the synchronizer by domestic and foreign research institutions, research on the friction coefficient between the friction pairs of the synchronizer has deepened. In reference [14], the dynamic model of the double-cone synchronizer was established based on the ADAMS software. On this basis, the effects of spring stiffness, the cone angle of friction ring, and the friction coefficient on shift force and the synchronization process were analyzed. Daniel Häggström analyzed the influence of temperature, speed difference, and shift force on the friction coefficient during synchronizer synchronization [15]. Sun Jun compared the influence of the friction coefficient on synchronizer performance and structure parameters under different inertia and speed difference [16]. Xi Junqiang conducted a bench test on the working process of the synchronizer. The results showed that the friction coefficient was not maintained at a constant value. In order to accurately reflect the real situation of the synchronization process, the friction coefficient must be considered [17].

In this paper, we study the influencing factors of the friction coefficient change of the synchronizer, and explore the quantitative variation law between the friction coefficient and each influencing factor, then develop the friction coefficient compensation control strategy to maintain the friction coefficient near the expected value and shorten the synchronization time. This is important to improve the shift quality.

\section{Analysis of the Influence of Friction Coefficient on Synchronous Performance}

\subsection{Evaluation Index of Synchronization Performance}

The shifting process should mainly follow the following principles: shorten shift synchronization time and drive interruption time as much as possible; the shifting process should avoid collisions and impacts to achieve smooth shifting; and minimize the sliding-friction work of the synchronization process to extend the synchronizer's service life. Therefore, the shift quality can be evaluated in terms of power interruption time, smoothness, and service life [18]. The locking ring synchronizer of automated mechanical transmission (a two-degrees-of-freedom direct-drive electromagnetic gearshift system) was the research object in this paper, as shown in Figure 1. The schematic diagram of the locking ring synchronizer is shown in Figure 1a, which consists of a synchromesh sliding sleeve, a splined hub, a synchronous ring, a target ring gear, and a locating slider [4,19].
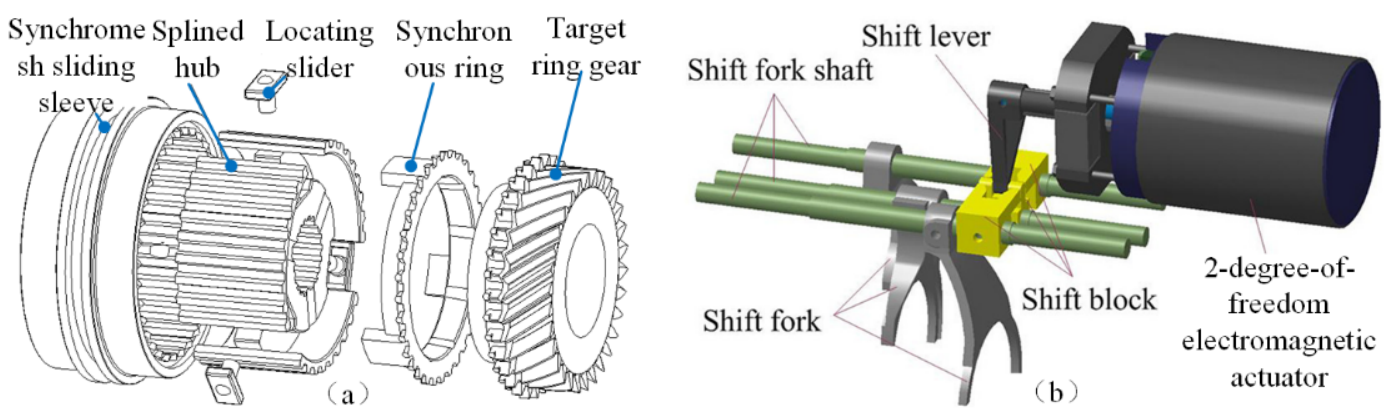

Figure 1. The scheme of the transmission: (a) the structure of the locking ring synchronizer; (b) two-degrees-of-freedom direct-drive electromagnetic gearshift system. 


\subsubsection{Shift Time}

The total time $t$ used for shifting is composed of the time $t_{1}$ taken for picking up the gear, the time $t_{2}$ used for pre-synchronization, the time $t_{s}$ used for synchronization, and the time $t_{4}$ taken for the combination - of these, the proportion of synchronization time $t_{s}$ is larger. The phase represented by the synchronization time has the greatest influence on the sliding-friction work and shock degree [20]. The following mainly studies the synchronization time $t_{s}$.

$$
t=t_{1}+t_{2}+t_{s}+t_{4}
$$

Establish the motion equation of the inertial mass at the input end as:

$$
J_{r} \frac{d \omega_{r}}{d t}=T_{f} .
$$

The synchronization time:

$$
\begin{gathered}
t_{s}=\int_{\omega_{e}}^{\omega_{c}} \frac{J_{r}}{T_{f}} d \omega_{r} ; \\
T_{f}=\frac{F_{s} \mu R}{\sin \alpha} ;
\end{gathered}
$$

where $T_{f}$ is the friction torque, $\mathrm{N} \cdot \mathrm{m} ; \mu$ is the friction coefficient; $F_{s}$ is the axial thrust, $\mathrm{N} ; R$ is the average radius of the friction cone, $\mathrm{m} ; J_{r}$ is the rotational inertia of the synchronizer's input, $\mathrm{Kg} \cdot \mathrm{m}^{2}$; $\omega_{r}$ is the angular speed of the synchronizer's input, $\mathrm{r} / \mathrm{min} ; \omega_{c}$ is the angular speed of the synchronizer's output, $\mathrm{r} / \mathrm{min}$; $\omega_{e}$ is the speed of the motor's input shaft, which is also the initial angular speed of synchronizer's input, $\mathrm{r} / \mathrm{min} ; \alpha$ is half the cone angle of the friction cone, rad.

\subsubsection{Shock Degree During Synchronous Phase}

The shock degree $(j)$ during the synchronous phase refers to the change rate of the angular speed of the synchronizer's input under the action of synchronous torque. In the synchronization phase, the smoothness of the system is closely related to the shock degree. The greater the shock degree, the more serious the vibration and noise will be. The shock degree during the synchronous phase is an important index to measure shift performance. Its calculation formula is,

$$
\left\{\begin{array}{l}
j=\frac{d\left(T_{\mathrm{f}}+T_{c}\right)}{J_{r} d t} \\
j_{\max }=\max (j)
\end{array},\right.
$$

where $T_{c}$ is the resistance moment of the transmission, $j_{\max }$ is the maximum shock degree during synchronous phase. It can be seen from Equation (5) that the change rate of synchronous torque determines the magnitude of the shock degree during synchronous phase. During the shift synchronization, the corresponding control strategy should be added to reduce the shock degree of this process, so as to improve the shift quality.

\subsubsection{The Sliding-Friction Work}

The synchronizer synchronizes between the synchronizing ring and the target ring gear, so that the components with different rotational speeds are synchronized, and friction heat generation is the most important implementation. The sliding-friction work is the work done by the friction torque on the relative angular displacement between the friction elements during the entire synchronization process. If excessive heat generates in the unit area during the sliding in a short time, it will cause irreversible damage to friction components and reduce the service life of synchronizer. The formula for calculating sliding-friction work $(W)$ is as follows:

$$
W=\int_{0}^{t_{s}} T_{f}\left|\omega_{c}-\omega_{r}\right| d t
$$


The evaluation indexes restrict each other during the shift synchronization process. In the process of pursuing an evaluation index improvement, it is necessary to coordinate with other indicators to ensure that the shift quality is comprehensively optimized.

\subsection{Simulation Analysis of Synchronization Performance}

The simulation model of a synchronizer in synchronization phase was built, and the influence of friction coefficient change between the synchronizer's friction pairs on synchronization performance was analyzed. According to the value range of the friction coefficient of the locking ring synchronizer's friction pair selected in this paper, the friction coefficient was set to $0.05,0.07$, and 0.09 in the simulation process, and the rotational inertia of the synchronized partial was $0.04 \mathrm{Kg} \cdot \mathrm{m}^{2}$. The influences of speed difference and friction coefficient on synchronization time were analyzed, as shown in Figure 2.

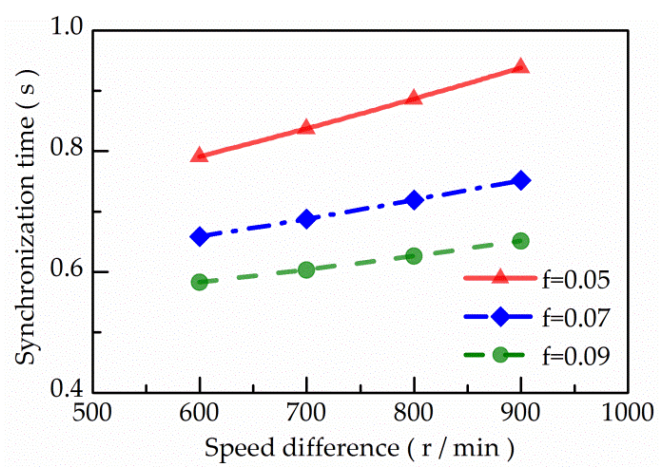

Figure 2. Synchronization time.

The larger the friction coefficient was, the shorter the synchronization time that was needed when the initial shifting speed difference was the same. With the increase in shifting speed difference, the effect of friction coefficient on synchronization time became more and more obvious. When the shifting speed difference was $600 \mathrm{r} / \mathrm{min}$ and the friction coefficient was 0.09 , the synchronization time was $0.58 \mathrm{~s}$. When the friction coefficient was 0.05 , the synchronization time was $0.79 \mathrm{~s}$. The difference between them was $0.21 \mathrm{~s}$. According to the simulation, the friction coefficient should be increased in order to shorten the synchronization time.

When the inertia of the synchronized part was $0.04 \mathrm{Kg} \cdot \mathrm{m}^{2}$, the influence of speed difference and friction coefficient on sliding-friction work was analyzed, as shown in Figure 3. The variation trend of sliding-friction work was basically the same under different shifting speed differences and friction coefficients. When the shifting speed difference was the same, the friction coefficient had no effect on the final value of sliding-friction work, but only affected the increase rate of sliding-friction work. The greater the friction coefficient was, the shorter the time it took for the sliding-friction work to reach the final value. When the shifting speed difference was $600 \mathrm{r} / \mathrm{min}$, the sliding-friction work consumed under the three friction coefficients was $78.96 \mathrm{~J}$. However, when the friction coefficient was 0.09 , the time needed to reach the final value of sliding-friction work was $0.21 \mathrm{~s}$ shorter than when the friction coefficient was 0.05 . When the final value of sliding-friction work was determined, the shorter the synchronization time was, the more heat generated per unit of time. Moreover, excessive heat generated per unit of time may have caused damage to the friction surface.

When the inertia of the synchronized part was $0.04 \mathrm{Kg} \cdot \mathrm{m}^{2}$, the influence of speed difference and friction coefficient on shock degree were analyzed, as shown in Figure 4. Since the maximum shock degree occurred at the initial stage in the synchronization process, the shock degree at the initial stage was intercepted for comparative analysis. It can be seen from the graph that the maximum shock degree decreased with the increase of friction coefficient when the shifting speed difference was the same. When the shifting speed difference was $600 \mathrm{r} / \mathrm{min}$, the maximum shock degree was $160.3 \mathrm{rad} / \mathrm{s}^{3}$ when the friction coefficient was 0.09 , and $290.4 \mathrm{rad} / \mathrm{s}^{3}$ when the friction coefficient was 0.05 . 


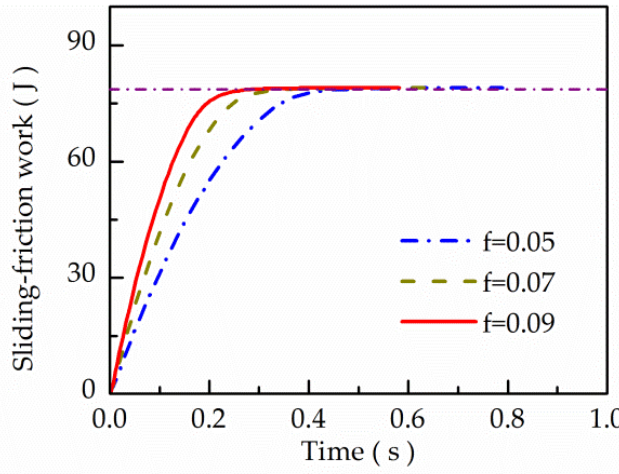

(a)

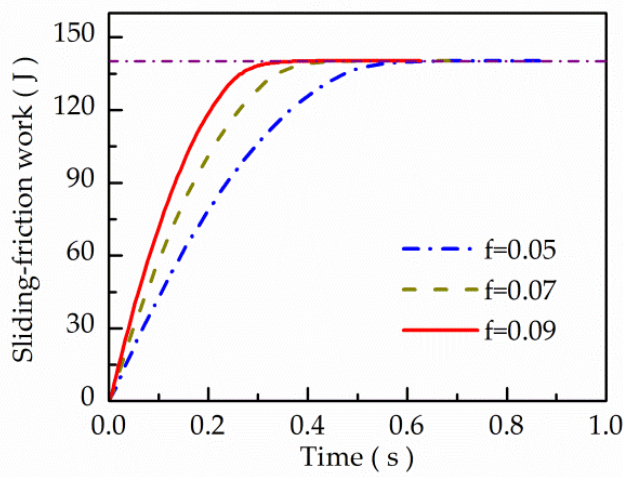

(c)

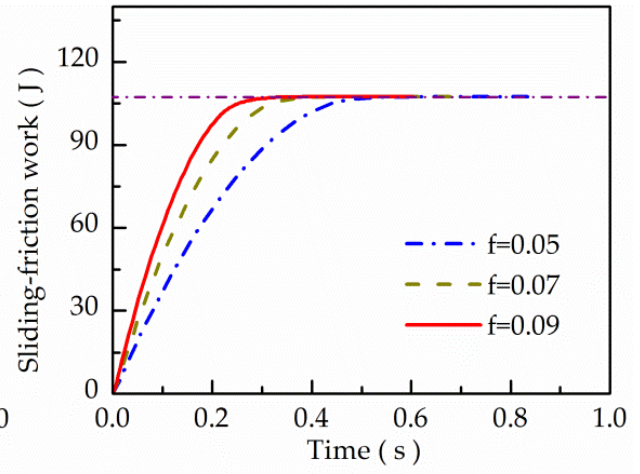

(b)

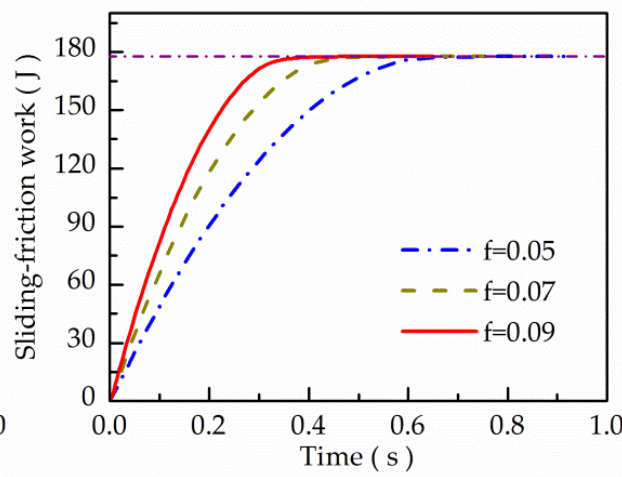

(d)

Figure 3. Sliding-friction work contrast: (a) shift speed difference $600 \mathrm{r} / \mathrm{min}$; (b) shift speed difference 700 r/min; (c) shift speed difference 800 r/min; (d) shift speed difference $900 \mathrm{r} / \mathrm{min}$.
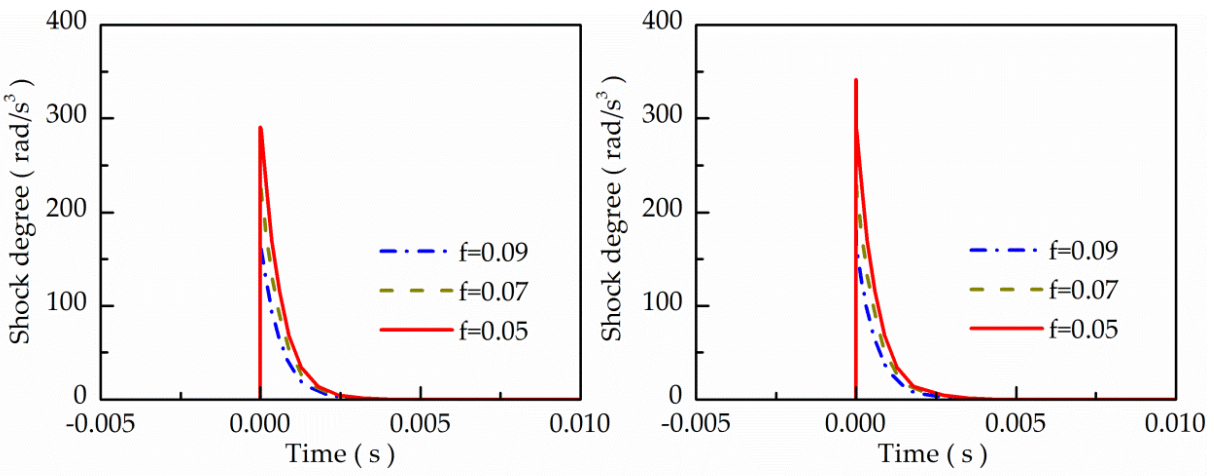

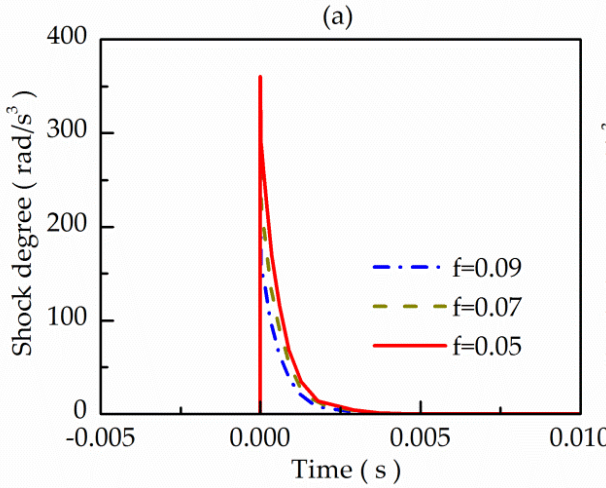

(c)

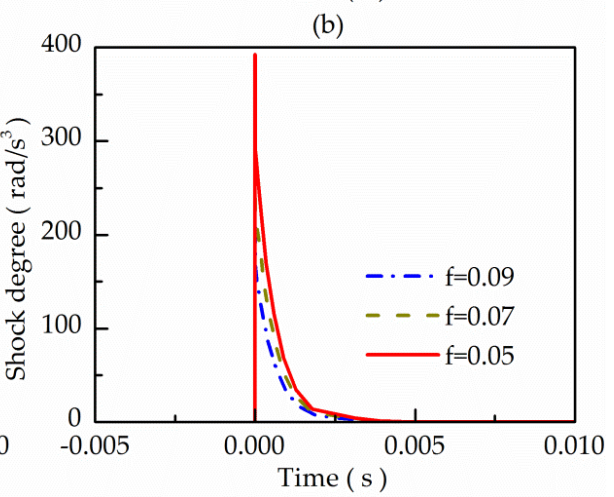

(d)

Figure 4. Shock degree contrast curve of synchronization early stage: (a) shift speed difference $600 \mathrm{r} / \mathrm{min}$; (b) shift speed difference $700 \mathrm{r} / \mathrm{min}$; (c) shift speed difference $800 \mathrm{r} / \mathrm{min}$; (d) shift speed difference $900 \mathrm{r} / \mathrm{min}$. 
In conclusion, with the increase in the friction coefficient, the synchronization time of the synchronizer decreased. The friction coefficient had no effect on the final value of sliding-friction work in the synchronous process, but it affected the increase rate of the sliding-friction work. At the same time, with the increase in friction coefficient, it took less time for the sliding-friction work to reach the final value, and the maximum shock degree at the initial stage decreased.

\section{Analysis of Friction Coefficient's Influencing Factors}

The premise of formulating the friction coefficient compensation control strategy is to obtain the quantitative relationship between the friction coefficient and its influencing factors. Thus, the friction test of the synchronization process must be performed first. A synchronizer test-control platform was built, and the friction coefficient MAP was obtained by collecting and calculating data during the test.

\subsection{The Synchronizer Test-Control Platform}

Based on the study of the synchronization process and mechanical analysis, combined with a modern electromechanical design concept, the synchronizer test-control platform was completed. The designed bench had the functions of variable voltage, variable speed, and constant output. The physical object of the synchronizer test-control platform is shown in Figure $5[19,21]$. The mechanical system of the test-control platform included inertia simulator, synchronizer component, shifting fork, displacement sensor (DS), and the connector that connected the components. The electronic control system included a driving device control system and three-phase asynchronous motor. The electromagnetic linear actuator (ELA) was controlled and driven by a driving device control system. The three-phase asynchronous motor output different speeds according to the driving requirements for the input shaft $[22,23]$. The inertia simulator on the test-control platform could simulate the range of rotational inertia from 0.04 to $0.09 \mathrm{~kg} \cdot \mathrm{m}^{2}$. The three-phase asynchronous motor was controlled by a frequency converter, and its maximum driving speed could reach $1200 \mathrm{r} / \mathrm{min}$. The electromagnetic linear actuator was used as a shift driving device, the maximum shift force was $1000 \mathrm{~N}$. The temperature was tested through an infrared camera (Optris PI450) with $-20 \sim 900{ }^{\circ} \mathrm{C}$ measuring range.

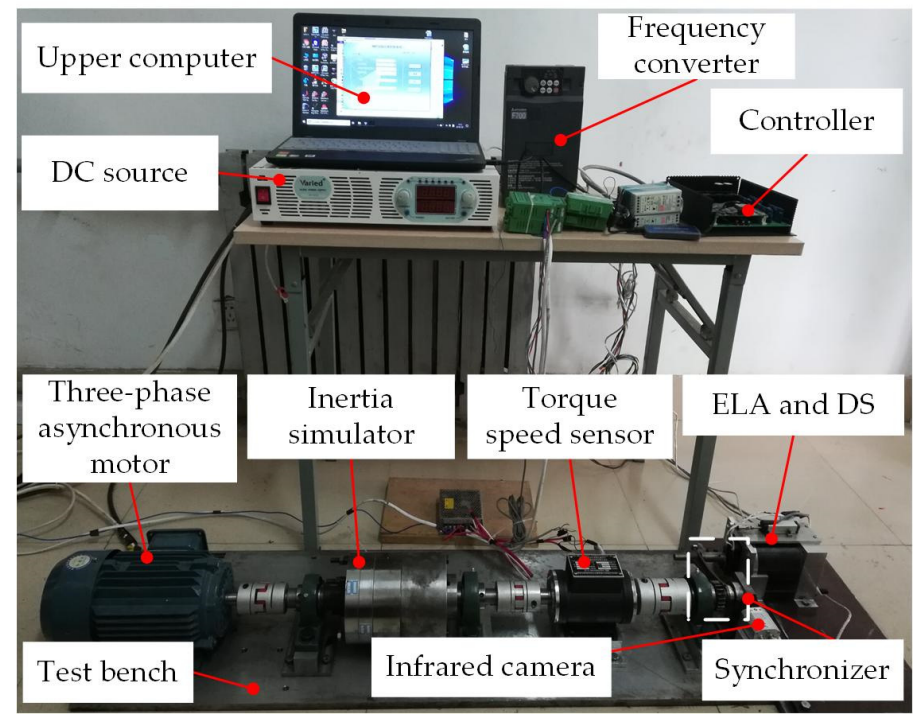

Figure 5. Synchronizer test-control platform.

\subsection{Quantitative Relationship between the Friction Coefficient and its Influencing Factors}

The results show that the friction coefficient between the friction cones of synchronizer is related to temperature, load, and speed difference between the synchronizer ring and target gear ring after the material and lubrication conditions of the synchronizer's friction cones are determined [15,24-27]. 
The premise of formulating the friction coefficient compensation control strategy was to obtain the quantitative relationship between friction coefficient and its influencing factors in the synchronization process. Firstly, the friction test of the synchronization process was carried out to obtain the sampling data of relevant parameters under different working conditions. The collected data was calculated and processed to obtain the control MAP, which was stored in the controller.

When the speed difference and shift force of the synchronizer were constant, the friction test between the synchronization ring and target gear ring was carried out. The controller collected state variables. In the same step, friction tests were carried out under different working conditions. The data was analyzed as typical when the synchronization time was $0.5 \mathrm{~s}$ under different working conditions. The operating conditions of the actual synchronization process were arbitrary, but the operating points of the MAP obtained by collecting data were limited and discrete, so the bicubic interpolation optimization method was needed to obtain the parameter values between the known operating conditions and the temperature and friction coefficient in the whole working condition could be obtained by the parameter values of finite working conditions [28]. After interpolation optimization, the friction coefficient MAP of $0.5 \mathrm{~s}$ synchronization time under different working conditions was obtained, as shown in Figure 6.

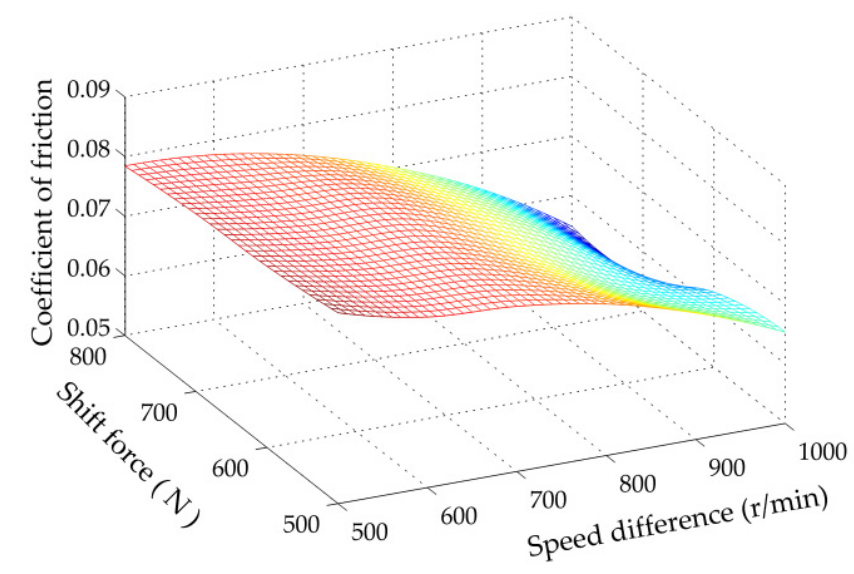

Figure 6. MAP diagram of friction coefficient after bicubic interpolation.

When the shifting speed difference was $600 \mathrm{r} / \mathrm{min}$ and the shifting force was $600 \mathrm{~N}$, the corresponding relationship of the friction coefficient changes with temperature was obtained, as shown in Figure 7. It can be seen from the graph that the friction coefficient decreased with the increase in temperature, and the friction coefficient decreased slowly when the temperature was low, and rapidly when the temperature was high. Due to the temperature rise, the specific surface energy and lattice resistance of the friction pair friction material changed. The temperature rise had an important influence on the mechanical properties and surface film formation of the friction material's surface layer, which made it easy to produce liquid lubrication film and resulted in the reduction in the friction coefficient.

Above is the corresponding relationship between the friction coefficient and speed difference, shift force and temperature when synchronization time was $0.5 \mathrm{~s}$. In order to obtain a quantitative relationship between the friction coefficient and the influencing factors in the whole working condition, a large number of tests were still needed, which will not be repeated here. The above work laid a foundation for formulating the friction coefficient compensation control strategy. 


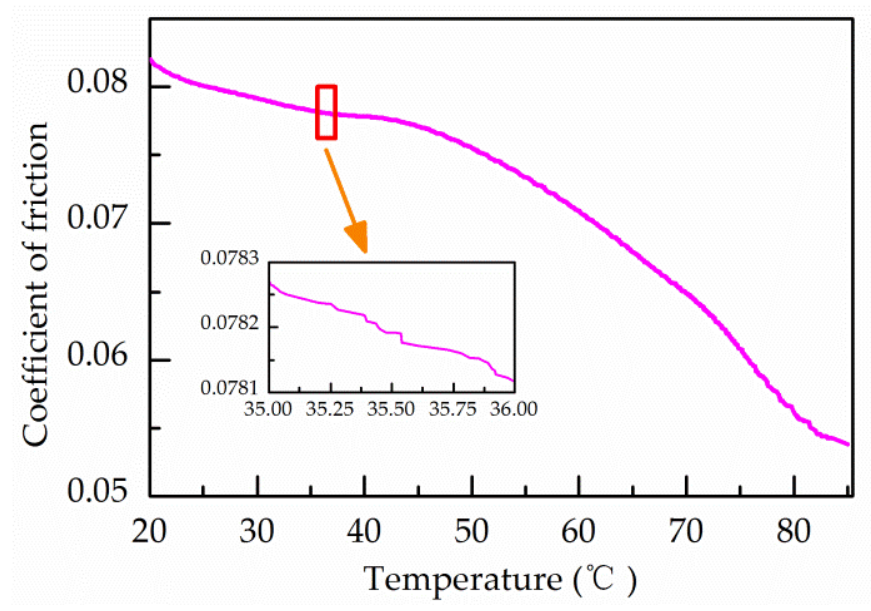

Figure 7. The corresponding diagram of temperature and friction coefficient.

\section{Friction Coefficient Compensation Control Strategy}

\subsection{Optimal Control}

In the synchronization process, the synchronization ring and the target gear ring are compressed by the shifting force, and the speed difference between them is eliminated by friction torque. The synchronization process takes a certain amount of time, the sliding-friction work and synchronization impact are also produced. The length of the synchronization time affects the shift interruption time. The magnitude of sliding-friction work affects the service life of the synchronizer, and the magnitude of shock degree affects the smoothness of synchronization process. The friction coefficient compensation control strategy of the synchronization process studied in this paper was bound to cause an increase in the shifting force. Increasing the shifting force would shorten the synchronization time, but due to the limitation of friction pair material and other two evaluation indexes, a greater shifting force would not be better. When the initial speed difference was fixed, shortening the synchronization time meant that the speed difference decreased faster and the shock degree was higher. However, in order to reduce the shock degree and reduce the shifting force, the synchronization time was prolonged and the synchronizer wear was increased. Therefore, in order to balance the evaluation indexes of each synchronization performance, it was necessary to limit the magnitude of the shifting force acquired by the friction coefficient compensation control strategy. Other evaluation indicators of synchronization performance were also optimized, while reducing synchronization time. According to the principle of minimum value, the optimal control method was adopted, and the evaluation indexes of the synchronization process of the synchronizer were optimized $[29,30]$.

The mathematical model of the shift synchronization process is expressed as:

$$
\left\{\begin{array}{c}
J_{n} \dot{\omega}_{r}=-\frac{T_{f}}{i_{g}} \\
J_{s} \dot{\omega}_{c}=T_{f}-\frac{T_{m}}{i_{n}}
\end{array},\right.
$$

where $J_{n}=\left[J_{c}+J_{m}\left(i_{g}, i_{n}\right)\right], J_{m}$ is the rotational inertia of the rotating part of the transmission equivalent to the input shaft, $J_{c}$ is the rotational inertia of clutch driven disk, $i_{g}$ is transmission ratio, $i_{n}$ is the final drive ratio, $T_{m}$ is the external resistance moment of the vehicle. The state vector is taken as:

$$
X=\left(\begin{array}{l}
x_{1} \\
x_{2} \\
x_{3}
\end{array}\right)=\left(\begin{array}{l}
\omega_{r} \\
\omega_{c} \\
\dot{\omega}_{c}
\end{array}\right) .
$$


The control vector is:

$$
Y=\left(\begin{array}{c}
y_{1} \\
y_{2}
\end{array}\right)=\left(\begin{array}{c}
T_{f} \\
\dot{T}_{f}
\end{array}\right)
$$

The state equation is established as:

$$
\left(\begin{array}{l}
\dot{x}_{1} \\
\dot{x}_{2} \\
\dot{x}_{3}
\end{array}\right)=\left(\begin{array}{lll}
0 & 0 & 0 \\
0 & 0 & 1 \\
0 & 0 & 0
\end{array}\right)\left(\begin{array}{l}
x_{1} \\
x_{2} \\
x_{3}
\end{array}\right)+\left(\begin{array}{cc}
-\frac{1}{J_{n} \cdot \dot{i}_{g}} & 0 \\
0 & 0 \\
0 & \frac{1}{J_{s}}
\end{array}\right)\left(\begin{array}{l}
y_{1} \\
y_{2}
\end{array}\right)+\left(\begin{array}{c}
0 \\
0 \\
-\dot{T}_{m} \\
\dot{i}_{n} \cdot J_{s}
\end{array}\right) .
$$

The maximum driving force produced by the electromagnetic linear actuator determined the range of synchronous moment. The maximum driving force of the actuator used in this paper was $1000 \mathrm{~N}$, so the maximum synchronous torque was $13.2 \mathrm{~N} \cdot \mathrm{m}$, that $0 \leq y_{1} \leq\left[y_{1}\right]=13.2 \mathrm{~N} \cdot \mathrm{m}$. $y_{2}$ stands for the change rate of synchronous torque, while the magnitude of the shock degree in the synchronous phase is determined by the change rate of synchronous torque. Therefore, the shock degree can be well limited by limiting the change rate of synchronous torque in synchronization phase. The constraint condition of calculating $y_{2}$ is $0 \leq y_{2} \leq\left[y_{2}\right]=1596$. In order to optimize each evaluation index, the optimal objective function is established as:

$$
J=\int_{t_{0}}^{t_{f}}\left[Q_{1} T_{s}\left(\omega_{c}-\omega_{s}\right)+Q_{2} j^{2}\right] d t
$$

where $J$ is the scalar function, $Q_{1}$ and $Q_{2}$ are the weight coefficients of sliding-friction work and shock degree, respectively. Due to the short shifting time, it is generally assumed that the external resistance torque of the vehicle is unchanged during this process, $\dot{T}_{m}=0$. Let $k=r / i_{n}$, the Hamiltonian function introduced based on the minimum principle is:

$$
H=Q_{1} y_{1}\left(x_{1}-x_{2}\right)+Q_{2}\left(\frac{k}{J_{s}} y_{2}\right)^{2}+\lambda_{1}\left(-\frac{y_{1}}{J_{n} \cdot i_{g}}\right)+\lambda_{2} x_{3}+\lambda_{3} \frac{y_{2}}{J_{s}}
$$

where $\lambda_{1}, \lambda_{2}$, and $\lambda_{3}$ are co-state variables. It is generally believed that the shifting force increases monotonously during the synchronization process, and the synchronous torque increases monotonously. Therefore, as long as the boundary of the optimal control variable is limited, the relative optimal trajectory and the optimal control result in the control domain range can be obtained. Computation is based on the necessary conditions to be met by minimum principles, and the intermediate steps are omitted.

$$
\lambda=\sqrt[3]{\frac{J_{s} Q_{1}}{2 Q_{2} k^{2}}}
$$

Thus,

$$
\begin{gathered}
y_{1}=-\frac{J_{s}}{2 Q_{2} k^{2}}\left[\left(\frac{\varepsilon_{1}}{\lambda}-\frac{\varepsilon_{2}}{\lambda^{2}}+\frac{2 \varepsilon_{3}}{\lambda^{3}}+\frac{\varepsilon_{2}}{\lambda} t-\frac{2 \varepsilon_{3}}{\lambda^{2}} t+\frac{\varepsilon_{3}}{\lambda} t^{2}\right) e^{\lambda t}\right. \\
\left.-\left(\frac{\varepsilon_{1}}{\lambda}-\frac{\varepsilon_{2}}{\lambda^{2}}+\frac{2 \varepsilon_{3}}{\lambda^{3}}+\frac{\varepsilon_{2}}{\lambda} t_{0}-\frac{2 \varepsilon_{3}}{\lambda^{2}} t_{0}+\frac{\varepsilon_{3}}{\lambda} t^{2}{ }_{0}\right) e^{\lambda t_{0}}\right]+y_{1}\left(t_{0}\right) \\
\varepsilon_{1}=-\frac{2 Q_{2} k^{2} \lambda T_{m}}{J_{s}} e^{-\lambda t_{0}}, \varepsilon_{2}=0, \varepsilon_{3}=0 .
\end{gathered}
$$

Finally, the optimal shifting force trajectory is obtained by the optimal control method:

$$
F^{*}= \begin{cases}\frac{-2 k^{2} Q_{2} \lambda T_{m} \sin \alpha}{J_{s} Q_{1} R \mu} e^{\lambda\left(t-t_{0}\right)}+y_{1}\left(t_{0}\right) \frac{\sin \alpha}{R \mu} & 0<F^{*}<F_{\max } \\ -F_{\max } & F^{*} \geq F_{\max } .\end{cases}
$$




\subsection{Compensation Control Principle}

The optimal shifting force trajectory $F^{*}$ was obtained by the optimal control method. Through the force controller, the voltage input to the electromagnetic linear actuator at different synchronization times was obtained, and the actuator output different shifting forces to the synchronizer according to different voltage values. During the synchronization process of the synchronizer, due to the difference in the rotation speed between the synchronizing ring and the target ring gear, the shifting force acted on the friction pair to generate the friction torque. The friction torque caused the temperature to rise during the synchronous speed difference, and the temperature accumulation caused the friction coefficient between the friction pairs to decrease. The real-time shifting force, the speed difference, and the temperature during the synchronization process were transmitted to the controller through the sensor [31-33]. The controller obtained the real-time friction coefficient $\mu$ by querying the relevant friction coefficient MAP. The real-time friction coefficient was compared with the expected friction coefficient $\mu^{*}$ to obtain the difference. The compensation controller calculated the compensation shifting force $F$ that needed to be output according to the difference. The compensation control force corrected the optimal control force in real time, and changed the force signal input to the force converter to maintain the friction coefficient of the friction pair of the synchronizer near the expected value. The feedback compensation control schematic is shown in Figure 8.

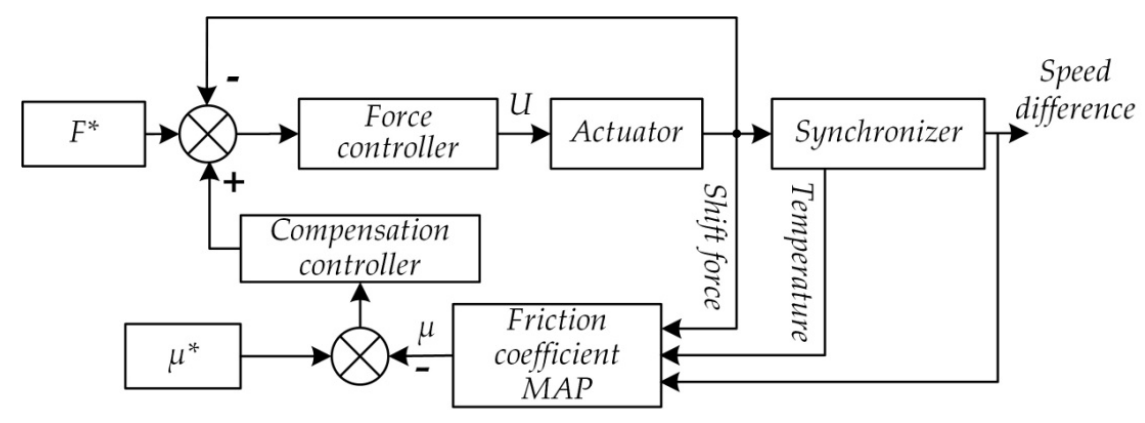

Figure 8. Principle of compensation control.

\section{Results and Analysis}

Based on the friction coefficient compensation control principle and the simulation model of the friction coefficient compensation control in the synchronizer synchronization process, the compensation control process was simulated and analyzed, and the synchronization performance index change after friction coefficient compensation was obtained. Because the friction coefficient compensation control system in the synchronization process of the synchronizer was a complex system with strong time-varying and multi-dimensional nonlinearity, the experiment was also carried out to verify the feasibility of the proposed friction coefficient compensation control strategy.

\subsection{Synchronization Time}

According to the literature and the performance of the synchronization loop, the expected friction coefficient of the synchronization process was set to be 0.08 , and the moment of inertia of the synchronized part was $0.04 \mathrm{Kg} \cdot \mathrm{m}^{2}$. The compensation control simulation and test were carried out under different initial shift speeds of the shift. The results of the simulation and test synchronization time with or without compensation were obtained, as shown in Figure 9. When the initial rotational speed difference of the shift was the same, the synchronization time used before the compensation in the test was longer than the compensation, and the regular was similar under different rotational speed differences. The shift speed difference was $600 \mathrm{r} / \mathrm{min}$, the synchronization time used in the pre-compensation test was $0.86 \mathrm{~s}$, and the synchronization time used after compensation was $0.74 \mathrm{~s}$. The compensation time required after compensation was shortened by $0.12 \mathrm{~s}$. The simulation results match the test results. The shift speed difference was $600 \mathrm{r} / \mathrm{min}$. The synchronization time used for the 
pre-compensation simulation was $0.70 \mathrm{~s}$. The synchronization time used after compensation was $0.62 \mathrm{~s}$. The compensation time required after compensation was shortened by $0.08 \mathrm{~s}$. It can be concluded that the synchronization time can be significantly shortened by compensating the friction coefficient, to ensure its maintenance near the expected value.

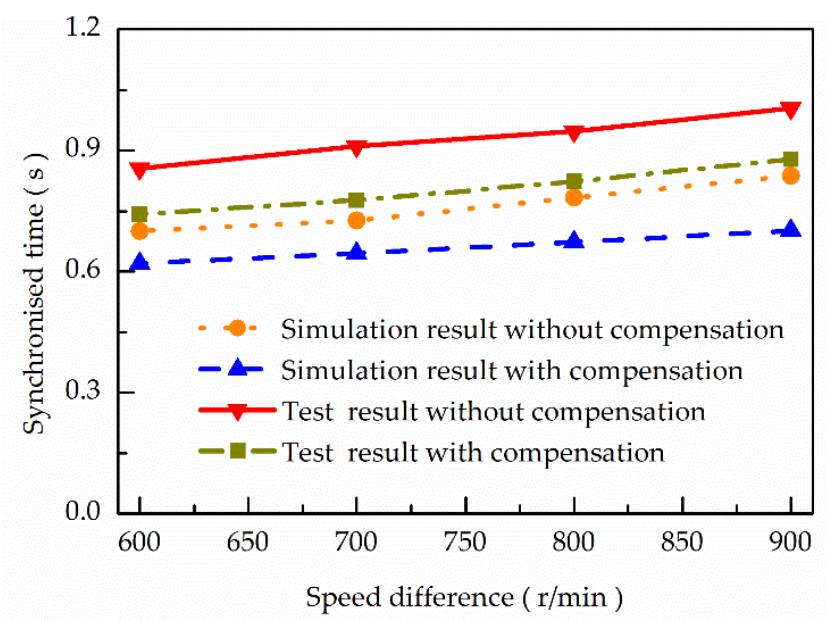

Figure 9. Synchronous time contrast with or without compensation.

\subsection{Shock Degree}

The initial speed difference was $600 \mathrm{r} / \mathrm{min}$, and the moment of inertia of the synchronized part was $0.04 \mathrm{Kg} \cdot \mathrm{m}^{2}$. The bench test of the synchronous process was carried out, and the comparison of the shock degree test before and after compensation was obtained, as shown in Figure 10. The initial synchronization phase had a relatively large shock degree before the compensation control, but the shock degree during the synchronization process was small and relatively stable. After the compensation control, the shock degree of the whole synchronization phase was relatively large, but the shock degree decreased with the continuous synchronization process. It can be seen from the synchronous phase shock degree calculation formula that the synchronous shock degree was related to the synchronous torque change rate during the synchronization of the synchronizer. The synchronous torque at this stage was the friction torque generated between the friction pairs of the synchronizer, and the magnitude of the friction torque was closely related to the shifting force acting on the shift fork. Therefore, the magnitude of the synchronous shock degree depended on the rate of change of the shifting force acting on the shift fork. It can be seen that in the process of compensating the friction coefficient of the synchronous process, the rate of change of the shifting force was gradually reduced, and the compensation control caused the shock degree in the synchronization process to become large.

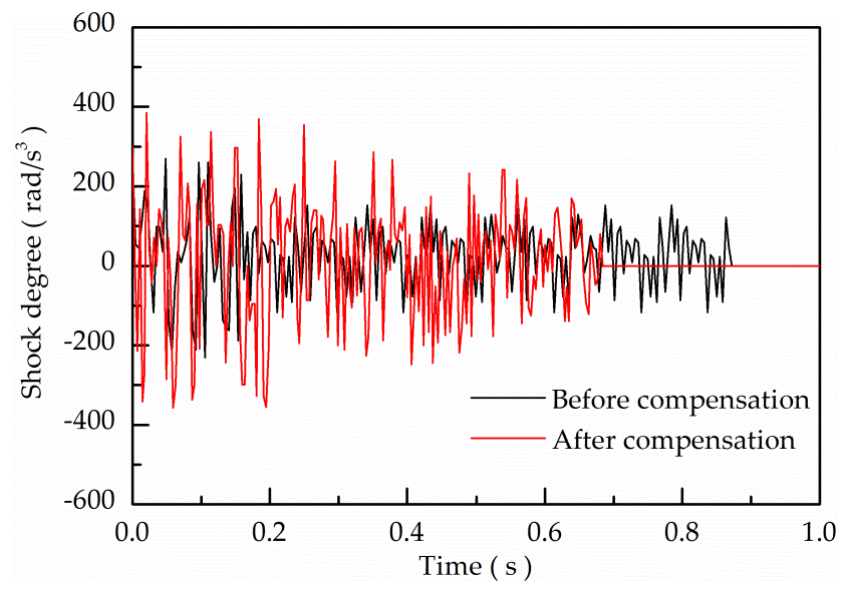

Figure 10. Comparison of shock degree before and after compensation. 


\subsection{Sliding-Friction Work}

When the initial speed difference of the shift was $600 \mathrm{r} / \mathrm{min}$, the expected friction coefficient of the synchronization process was 0.08 . The simulation results of sliding-friction work before and after compensation were compared, as shown in Figure 11.

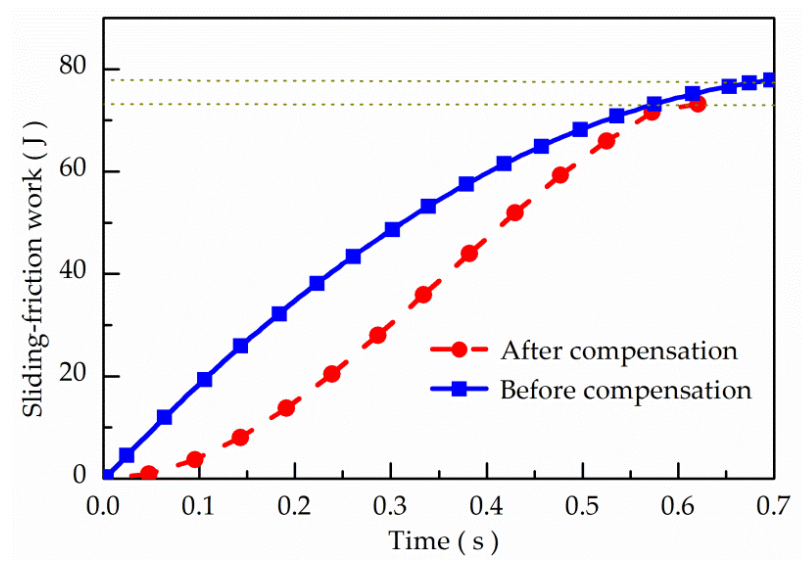

Figure 11. Comparison of sliding-friction work before and after compensation.

The final value sliding-friction work after the synchronization phase of the synchronizer was less than that before the compensation, and the time to reach the final value after compensation was shorter than that before compensation. It can be seen from the trend of the sliding-friction work that after the compensation, the sliding-friction work growth in the early stage was slow, and the growth in the later stage was fast, while that before the compensation sliding-friction work had a fast growth in the early stage and a slow growth in the later stage. The shifting speed difference was $600 \mathrm{r} / \mathrm{min}$, the final value sliding-friction work in the before compensation phase was $77.89 \mathrm{~J}$, and the final value sliding-friction work in the after compensation was $73.25 \mathrm{~J}$. After the compensation, the final sliding-friction work value was reduced by $4.64 \mathrm{~J}$. It can be seen from the comparison of the sliding-friction work before and after compensation that the sliding-friction work can be reduced by compensating the friction coefficient.

\subsection{Friction Coefficient}

When the initial shift speed difference was $600 \mathrm{r} / \mathrm{min}$, the moment of inertia of the synchronized part was $0.04 \mathrm{Kg} \cdot \mathrm{m}^{2}$. After compensation, the test and simulated friction coefficient results were compared, as shown in Figure 12.

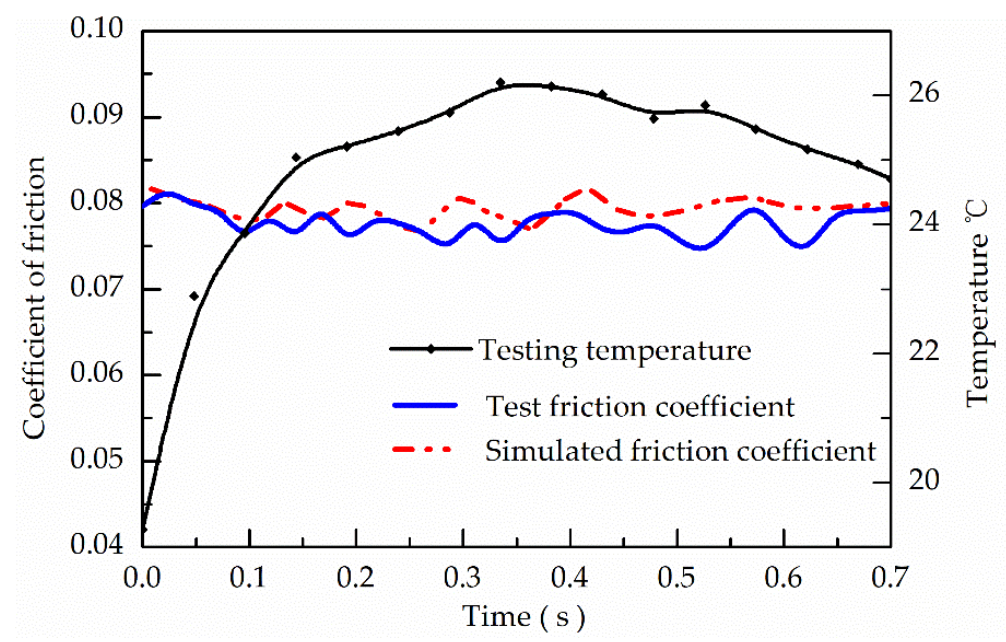

Figure 12. Friction coefficient after compensation. 
In the experiment, the temperature increased first and then decreased. At the initial stage of friction, the energy of heat flux density input was greater than the energy of heat conduction and convective heat transfer taken away, and the temperature increased speedily. With the decrease in speed difference at both ends of the synchronizer, the heat flux and convective heat transfer coefficient of the friction cone decreased gradually. When the energy of heat flux density input was equal to the energy taken away, the temperature of the synchronizer ring friction cone reached the maximum. As the speed difference at both ends of the synchronizer decreased continuously, the temperature of the synchronizer ring friction surface reduced gradually until the end of synchronization process. It can be seen from Figure 12 that after the compensation control, the friction coefficient simulation and test values during the synchronization of the synchronizer had been fluctuating around the expected value, which satisfied the expected control target. However, the fluctuation coefficient of the friction coefficient test value during the synchronization process was larger than expected-the expected friction coefficient was 0.08 , and the friction coefficient obtained by the fitting was slightly lower than 0.08 . Therefore, it was necessary to carry out a large number of tests in the later stage, and a better friction coefficient compensation control of various MAP, and consider various errors in the compensation control process to obtain a better control performance.

By comparing the synchronization time, shock degree, sliding-friction work, and friction coefficient before and after the synchronization of the synchronizer, it can be concluded that the compensation control strategy can maintain the friction coefficient of the synchronization near the expected friction coefficient. The synchronization time was shortened, and the sliding-friction work consumed in the synchronization phase was reduced. It is only the shock degree in the synchronization phase that was slightly larger, but the maximum value of the shock degree in the synchronous phase was smaller than the shock degree in the uncompensated phase. It can be seen from the performance evaluation indicators of these synchronization stages that the friction coefficient compensation control strategy can have a good effect. From the perspective of shortening the synchronization time, the reduction in the synchronization time can reduce the shift interruption time, which is crucial for shift quality.

\section{Conclusions}

The quantitative relationship between the friction coefficient and its influencing factors was studied. Based on the quantitative analysis and an optimal shift force control method, a compensation control strategy for the friction coefficient was established and verified. The main results can be summarized as follows:

1. After the friction coefficient compensation control, the friction coefficient was maintained near the expected value of 0.08 , which verified the effectiveness of the friction coefficient compensation;

2. Under the compensation control strategy based on an optimal shift force control method, the shifting speed difference of the synchronous time was shortened by nearly $0.12 \mathrm{~s}$ and the sliding friction was reduced by $4.64 \mathrm{~J}$ under the experimental conditions. The shift quality was improved significantly.

Author Contributions: Conceptualization, H.Y. and Z.X.; Data Curation, J.Y.; Formal Analysis, H.Y.; Funding Acquisition, W.G.; Methodology, H.Y.; Project Administration, Z.X.; Resources, Z.X. and J.Y.; Supervision, Z.X.; Validation, H.Y. and W.G.; Writing-Original Draft, H.Y. and M.L.; Writing—Review \& Editing, J.Y. and M.L.

Funding: This work is supported by the National Natural Science Foundation of China (51875290); Shandong Provincial Natural Science Foundation, China (ZR2019MEE049); Shandong Provincial Key Research and Development Project, China (2019GGX104018).

Conflicts of Interest: The authors declare no conflict of interest.

\section{References}

1. Wang, S.L.; Ling, C.; Xi, J.Q. Test and simulation of friction factor change in synchronizer working process. Automob. Technol. 2015, 7, 32-34. 
2. Marcus, S. Einfluss der Temperaturverteilung auf das Tribologishe Verhalten von Synchronisierung. Ph.D. Thesis, Leibniz Universität Hannover, Hannover, Germany, 2001.

3. Zhou, Y.; Chang, S. A Model-Assisted Reduced-Order ESO Based Cascade Controller for Sensorless Control of Independent Gear-Shifting Actuators. Appl. Sci. 2017, 7, 1283. [CrossRef]

4. Li, B.; Ge, W.; Yu, X.; Shao, S.; Liu, H. Innovative design and gearshift control for direct-drive electromagnetic gearshift system equipped with servo synchronizer. Proc. Inst. Mech. Eng. Part D J. Automob. Eng. 2019, 233, 1115-1124. [CrossRef]

5. Jo, C.; Ko, J.; Yeo, H.; Yeo, T.; Hwang, S.; Kim, H. Cooperative regenerative braking control algorithm for an automatic-transmission-based hybrid electric vehicle during a downshift. Proc. Inst. Mech. Eng. Part D J. Automob. Eng. 2012, 226, 457-467. [CrossRef]

6. Zhao, E.H.; Ma, B.; Li, H.Y. Study on the high temperature friction and wear behaviors of cu-Based friction pairs in wet clutches by pin-on-disc tests. Adv. Mater. Sci. Eng. 2017, 1-8. [CrossRef]

7. Beer, S.; Ginosar, R. A model for supply voltage and temperature variation effects on synchronizer performance. IEEE Trans. Very Large Scale Integr. Syst. 2015, 23, 2461-2472. [CrossRef]

8. Chen, Z.G.; Shao, Y.M.; Lim, T.C. Non-linear dynamic simulation of gear response under the idling condition. Int. J. Automot. Technol. 2012, 13, 541-552. [CrossRef]

9. Kovacevic, I.; Djelosevic, M.; Tepic, G.; Milisavljevic, S. Influential parameters and numerical simulation of heat generated in the process of friction stir welding. Mater. Sci. 2016, 22, 348-353. [CrossRef]

10. Li, M.; Khonsari, M.; Lingesten, N.; Marklund, P.; McCarthy, D.M.C.; Lundin, J. Model validation and uncertainty analysis in the wear prediction of a wet clutch. Wear 2016, 364, 112-121. [CrossRef]

11. Pär, M.; Roland, L. Wet clutch friction characteristics obtained from simplified pin on disc test. Tribol. Int. 2008, 41, 824-830.

12. You, G.G.; Yang, C.; Li, J.N. Parameter optimization and working process analysis of locking ring synchronizer. Mech. Transm. 2016, 40, 73-76.

13. Huang, H.; Nowoisky, S.; Knoblich, R. Modeling and testing of the hydro-mechanical synchronization system for a double clutch transmission. In Proceedings of the 9th International Modelical Conference, Munich, Germany, 3-5 September 2012; pp. 287-294.

14. Li, J.; Luo, F.; Luo, Y. Sensitivity Analysis on a Synchronization Mechanism for Manual Transmission Gearbox. SAE Tech. Pap. 2014, 1, 1768. [CrossRef]

15. Häggström, D.; Nyman, P.; Sellgren, U.; Björklund, S. Predicting friction in synchronizer systems. Tribol. Int. 2016, 97, 89-96. [CrossRef]

16. Sun, J.; Cao, L.T. Analysis of the influence of variable friction coefficient on synchronizer performance. Agric. Equip. Veh. Eng. 2016, 54, 19-23.

17. Ling, C.; Xi, J.Q. Simulation research on synchronizer working process. J. Automot. Eng. 2015, 5, 9-15.

18. Ge, A.L.; Shen, B. Research on AMT shift quality. Automot. Technol. 2003, 3, 43-45.

19. Yan, H.; Xu, Z.P.; Yuan, J.T.; Liu, L.; Tan, C.; Ge, W. Friction Characteristics of Synchronization Process Based on Tribo-Thermodynamics. Adv. Mater. Sci. Eng. 2018, 1-9. [CrossRef]

20. Lin, S.S. Research on Shift Control Technology of Mechanical Automatic Transmission Based on Direct Drive. Ph.D. Thesis, Nanjing University of Science and Technology, Nanjing, China, 2013.

21. Hao, J.J.; Ding, X.H.; Wei, J.W. Design of test bench for transmission efficiency of AMT actuator. Appl. Mech. Mater. 2013, 307, 79-83. [CrossRef]

22. Liu, M.Y.; Tan, C.; Li, B. Experimental study on frictional characteristics of friction cone in synchronous process of lock-ring synchronizer. Mech. Des. Res. 2019, 35, 154-156.

23. Li, B.; Tan, C.; Wang, G.; Ge, W.; Sun, B. Sensorless landing control strategy of bistable permanent magnet actuator. Math. Probl. Eng. 2019, 1-11. [CrossRef]

24. Marklund, P.; Mäki, R.; Larsson, R.; Höglund, E.; Khonsari, M.; Jang, J. Thermal influence on torque transfer of wet clutches in limited slip differential applications. Tribol. Int. 2007, 40, 876-884. [CrossRef]

25. Brown, G.; Walker, G.M.; Friend, C. Understanding MTF additive effects on synchronizer friction. Int. J. Fuels Lubr. 2012, 5, 447-458. [CrossRef]

26. Xie, B.Y.; Zhang, Q.; Lu, Y.F. Study on friction factor of emergency brake friction plate. Trans. Chin. Soc. Agric. Mach. 2006, 37, 33-35.

27. Wang, Y.; Wang, B.; Guo, R. Research on variable working condition control of vehicle electric control system based on MAP. Automob. Technol. 2017, 3, 42-46. 
28. Han, B.Y.; Chu, J.W. The dynamic and economical property of oxygen-enriched intake air gasoline engine based on MAP. Int. J. Digit. Content Technol. Appl. 2013, 7, 812-820.

29. Chai, B.B.; Wu, S.F.; Zhang, J.W. Optimal control of shifting process of electric drive two-speed automatic transmission. J. Shanghai Jiao Tong Univ. 2018, 52, 658-665.

30. Zhao, H.Q.; Zhao, W.; Wang, J.F. Optimal control of shift synchronization process of mechanical automatic transmission of tracked vehicles. J. Armored Force Eng. Inst. 2018, 32, 51-56.

31. Hu, T.H.; Yeh, C.J. Design of control logic and compensation strategy for electric power steering systems. In Proceedings of the 2008 IEEE Vehicle Power and Propulsion Conference, Harbin, China, 3-5 September 2008.

32. Kawai, K.; Zanma, T.; Ishida, M. Simultaneous vibration suppression control for permanent magnet synchronous motor using repetitive control and feed forward compensation. IEEE Trans. Ind. Appl. 2007, 127, 747-754. [CrossRef]

33. Zheng, H.Y.; Liu, H.Z.; Zong, C.F. Compensation torque algorithm for electric power steering system of puncture cars. J. Jilin Univ. 2012, 42, 521-526.

(C) 2019 by the authors. Licensee MDPI, Basel, Switzerland. This article is an open access article distributed under the terms and conditions of the Creative Commons Attribution (CC BY) license (http://creativecommons.org/licenses/by/4.0/). 